\title{
'NÉ MENDICANTI NÉ POVERI': \\ LA LIBERTÀ NELLE UTOPIE ITALIANE DEL RINASCIMENTO
}

\section{Cristina PerissinotTo}

Riassunto: Questo saggio analizza il problema della libertà in utopia con l'aiuto di un apparato filosofico di tradizione principalmente italiana e anglosassone, ove il discorso sulla libertà è sempre stato centrale. Alla luce dell'utopia eponima, quella di Thomas More, si discute il ruolo che alla libertà venne destinato nelle utopie italiane del Rinascimento. Si indica una strada per risolvere l'aporia che vede libertà e utopia come concetti contrapposti, dimostrando che per connetterli occorre calarsi in una realtà affatto diversa da quella contemporanea, e studiare l'universo teorico in cui la libertà rinascimentale convive con l'idea di utopia, mondo migliore, città ideale.

Questo articolo opera un confronto sul tema cruciale delle utopie italiane scritte tra Cinquecento e Seicento, usando come termine di paragone l'utopia di Thomas More, che iniziò tale genere letterario e fu pubblicata nel 1516.

L'importanza del concetto di libertà in utopia è duplice: da un lato la riflessione utopistica che sorge nel Rinascimento e nella splendida stagione delle utopie italiane tra Cinquecento e Seicento rappresenta il sentire degli intellettuali della società di allora sulla libertà. Dall'altro lato le utopie rinascimentali scritte in Italia servirono anche come insegnamento per i governanti coevi e futuri. Molte di esse furono prodotte e pubblicate come specula principum e vennero apprezzate e studiate nei secoli successivi. Insieme all'utopia di Thomas More, esse insegnarono al mondo a "immaginare" la città e la società e a concepire un mondo migliore.

Il concetto di libertà è fondamentale per comprendere sia l'utopia italiana, che il mondo desiderato nel Rinascimento. Tale concetto è anche al centro della riflessione di un vasto numero di discipline tra cui la scienza politica, i cultural studies, il discorso di genere, gli studi rinascimentali e, per la specificità di questo articolo, gli studi di italianistica. Il corpus delle utopie italiane analizzate in questo articolo include utopie note e meno note, da Campanella ad Antonfrancesco Doni, da Ludovico Zuccolo a Mambrino Roseo. Questo articolo intende analizzare il problema della libertà nelle utopie italiane tramite l'uso di un apparato filosofico e politico, senza però perdere di vista il contesto storico. 


\section{Importanza del concetto di libertà nelle utopie}

Immaginiamo di leggere sui giornali la notizia di una remota comunità che strappa i neonati alle braccia delle loro madri per allevarli in collegi comunitari, nei quali i bambini presto dimenticano i genitori. Immaginiamo anche che ciò sia fatto allo scopo di eliminare il sentimento d'amore, che si apprende con l'attaccamento al seno materno. Penseremmo di avere a che fare con una comunità di rara crudeltà, che viola diritti umani fondamentali e lede in modo criminale la libertà delle famiglie e dei minori. Tale comunità metterebbe a rischio il benessere degli individui e l'istituzione della famiglia. Sebbene una comunità siffatta non esista né a memoria d'uomo sia mai esistita, se non sulla carta, tale agghiacciante scenario rappresenta solo uno dei mondi possibili immaginati dagli utopisti rinascimentali per realizzare uno stato più giusto ed efficiente.

Nonostante l'inquietudine che potrebbe destare tale scenario, il pensiero utopistico rappresentò un momento di grande apertura nella vita intellettuale del Rinascimento, soprattutto nell'Italia cinque-secentesca, che produsse un numero notevole di pregevoli utopie letterarie. Tra le questioni fondamentali nello studio delle utopie non vi è solo la loro realizzabilità, ma le implicazioni che derivano dal concepire mondi siffatti; non la loro realizzabilità, ma la loro possibilità. Infatti la possibilità nelle utopie è sempre stata presente, mentre la loro realizzabilità ha una sua origine storica, legata ai confini del mondo conosciuto. Come scrive Reinhart Koselleck, nel Rinascimento "il globo terrestre era conosciuto nella sua configurazione sferica, nella sua finitezza e nella finitezza delle sue superfici. Dunque non era più possibile situare utopie in qualsiasi luogo del pianeta, poiché i navigatori potevano sapere se l'isola di cui un utopista parlava esisteva davvero." Quando il mondo fu completamente conosciuto avvenne il passaggio dell'utopia dallo spazio al tempo, vale a dire la sua collocazione nel futuro divenne condizione necessaria per concepire l'universo utopico ${ }^{1}$.

1 "il globo terrestre era conosciuto nella sua configurazione sferica, nella sua finitezza e nella finitezza delle sue superfici. Dunque non era più possibile situare utopie in qualsiasi luogo del pianeta, poiché i navigatori potevano sapere se l'isola di cui un utopista parlava esisteva davvero. Di conseguenza, nel Settecento le utopie si erano già trasferite sulle stelle o erano sprofondate sottoterra. Furono immaginati scenari utopici celesti o sotterranei. Oppure si ritornava al passato, dove regnavano condizioni da Paese di Bengodi per le famiglie o esistevano società perfettamente trasparenti. Il pianeta era dunque occupato. La via d'uscita che ora si apriva era il futuro. [...] Da allora, quasi tutte le utopie sono orientate verso il futuro. In questo modo muta la struttura di un'utopia.” Koselleck, Il vocabolario della modernità, 142 . 
Nel Cinquecento, le utopie erano di solito legate al racconto di viaggio. Se non ci s'interroga molto sull'attualità/possibilità e realizzabilità di utopie già realizzate ci s'interroga ancor meno su un problema cruciale dell'universo utopistico, quello della libertà.

Questo saggio inquadra filosoficamente il problema della libertà in utopia, con l'aiuto di un apparato teorico di tradizione sia italiana che anglosassone, ove il discorso sulla libertà è centrale. Ci si propone di interrogare le utopie italiane alla luce del loro modello principe, l'Utopia di Thomas More, sul ruolo che alla libertà venne destinato nelle utopie italiane del Rinascimento. Si dimostrerà che al tempo l'idea di libertà presentava caratteristiche radicalmente diverse dalla nostra concezione in età contemporanea. Si dimostrerà che per comprenderla occorre calarsi in un sistema di valori e in un apparato teorico che sono radicalmente diversi dal nostro. Si dimostrerà che il mondo delle utopie rinascimentali, in particolar modo quelle italiane, presenta una serie di scarti teorici che costringono lo studioso a guardare la realtà con altri occhi, nei quali la libertà assume carattere e connotazioni diverse. Se ciò non avviene il mondo delle utopie rinascimentali viene frainteso, e visto come eternamente aporetico, basato cioè sull'insolubile contraddizione tra giustizia e eguaglianza della società da un lato e limitazioni alla libertà individuale dall'altro.

La discussione sulle utopie rinascimentali e sulla loro apparente restrizione della libertà non è un esercizio fine a se stesso, dato che i due concetti di libertà e utopia vengono oggi percepiti come contraddittori. In base a tale considerazione non si comprende appieno come il pensiero politico rinascimentale abbia potuto concepire modelli di società migliore senza tener presente le molte restrizioni alla libertà individuale. Non è possibile d'altra parte fermarsi alla constatazione di tale contraddizione, per quanto interessante e gravida di considerazioni proficue. Il fatto che nel Rinascimento si sia (ri)scoperta la formula dell'utopia (così come proposta da Thomas More) e che si sia continuato a scriverne nonostante la cogente contraddizione tra mondo migliore e libertà fa riflettere sulle modalità del pensiero politico agli albori della modernità.

Perché di pensiero politico, oltreché di opere letterarie, si trattava. Essendo le utopie del Rinascimento considerate produzioni non solo letterarie ma anche politiche e pratiche, i lettori coevi non le lessero mai come opere di pura immaginazione. Le collocarono invece nella sfera della politica, e di conseguenza nel campo del ragionamento pratico ${ }^{2}$, anche se non necessariamente passibili di applicazioni pratiche.

2 È ciò che sostiene Richard Ashcraft a proposito della teoria politica di John 
Il discorso dell'utopia rinascimentale va quindi affrontato anche nei suoi aspetti di filosofia pratica. Se le utopie rinascimentali vennero, in toto $\mathrm{o}$ in parte, lette e apprezzate alla luce della politica del tempo, è necessario discutere come vennero affrontati i temi specifici del pensiero politico, primo fra tutti il concetto di libertà e quello relativo di oppressione.

La libertà dei cittadini è il nodo più importante di ogni società, al punto che nessuna società immaginaria o reale verrebbe considerata desiderabile se la libertà individuale fosse apertamente minacciata. D'altra parte, nelle utopie scritte in Italia nel Rinascimento, quelle che imitarono l'opera eponima di Thomas More, la libertà personale degli individui era variamente minacciata dalle leggi scritte e non scritte della stessa società, vale a dire dalle norme fissate dallo scrittore di utopie.

\section{Uomini liberi e schiavi per natura}

Nel pensiero critico sull'utopia non sono mancate interpretazioni atte a minimizzare alquanto la crudeltà della schiavitù nell'universo utopico, ma nelle utopie rinascimentali la schiavitù è presente e in stretto collegamento con il pensiero classico, da Aristotele alla tradizione giuridica romana ${ }^{3}$, poiché faceva parte dell'universo economico di Roma e delle colonie.

Thomas More scrive che gli utopiani non considerano schiavi i prigionieri di guerra, a meno che non siano stati essi stessi a scatenarla; tuttavia essi riducono in schiavitù

quelli tra i proprii concittadini che sono stati ridotti in servitù a causa di un delitto commesso, oppure (ed è il caso di gran lunga più frequente) quelli che in città straniere in seguito ai loro crimini sono stati condannati a morte. ${ }^{4}$

Locke, ragionamento che si può estendere anche ad altri ambiti del pensiero politico. "Locke pensava che la teoria politica significasse ragionare di politica."("Locke believed that political theorizing was an exercise in political reasoning.") Ashcraft, "Locke’s Political Philosophy," 226.

3 Per Aristotele si veda il celebra passo della Politica, I, 5 "Perciò la natura vuol segnare una differenza nel corpo dei liberi e degli schiavi: gli uni l'hanno robusto per i servizi necessari, gli altri eretto e inutile a siffatte attività, ma adatto alla vita politica [...]. Dunque, è evidente che taluni sono per natura liberi, altri schiavi, e che per costoro è giusto essere schiavi." Per il diritto romano si veda Paola Biavaschi, "La questione della schiavitù nel diritto romano, alla luce del mito di Atlantide e della città ideale."

${ }^{4}$ More, Utopia, 247. 
Cosimo Quarta, studioso e ammiratore di Thomas More, considera la schiavitù degli stranieri in Utopia come "assimilabile ai moderni emigran$t i$ "s. Tale visione è anche supportata da certa storiografia anglosassone, che vede la schiavitù in More come una "metafora dell'autodisciplina"6. Non mancano però le voci altamente critiche di questo aspetto dell'utopia, tra cui quella di Shlomo Avineri. Il suo articolo su guerra e schiavitù in Utopia risuona di indignazione per le pratiche schiaviste nell'Utopia di Thomas More ${ }^{7}$.

Al contrario di More, che dedica alla schiavitù un intero capitolo, non vi è, nelle utopie italiane del Rinascimento, una trattazione specifica sugli schiavi, come non vi era nella Repubblica di Platone, dove la schiavitù era una pratica normale.

Nel Rinascimento il discorso sulla libertà riguardava sia la libertà politica che il libero arbitrio. Si tendeva però ad ignorare il problema della libertà degli schiavi perché a quel punto il mondo occidentale si basava economicamente sullo schiavismo. Il Rinascimento infatti riportò in auge lo schiavismo e non percepì la libertà degli uomini liberi come contraddittoria rispetto all'esistenza degli schiavi ${ }^{8}$. La tratta degli schiavi nel mondo occidentale divenne fondamentale per mantenere lo stile di vita degli uomini liberi甲.

Pur non essendo schiavi per natura, gli abitanti delle varie utopie rinascimentali vedono ristretta la propria libertà in vario modo, con una sola

5 Quarta, Tommaso Moro, 223.

6 Johnson, More's Utopia: Ideal and Illusion, 107. Citato anche in Quarta, Tommaso Moro, 222.

7 Avineri. "War and Slavery in More's Utopia," 260-290. Si vedano anche: Grele, "Relations économiques et rapports sociaux," 19-32 ; Lourde Reyes, "La Utopía y el nuevo mundo," 95-101.

8 Quilligan "Freedom, Service and the Trade in Slaves," 213-234.

9 Si veda a questo proposito David Brion Davis: "la contraddizione inerente alla schiavitù umana ha sempre generato una forma di pensiero dualistico, ma a partire dal sedicesimo e diciassettesimo secolo gli Europei erano arrivati al più grande dualismo, la fondamentale divisione tra sempre maggiore devozione verso la libertà in Europa, e un sistema mercantile in espansione basato sul lavoro dei Negri in America." ("The inherent contradiction of human slavery had always generated dualisms in thought, but by the sixteenth and seventeenth centuries Europeans had arrived at the greatest dualism of all - the momentous division between an increasing devotion to liberty in Europe and an expanding mercantile system based on Negro labor in America.") Davis, The Problem of Slavery in Western Culture, 108. 
eccezione, che vedremo subito ${ }^{10}$.

\section{L'abbazia di Thélème e la libertà assoluta}

Non in tutte le utopie rinascimentali la libertà individuale è soggetta a limitazioni. Nell'abbazia di Thélème ad esempio, inventata da Rablais in Gargantua et Pantagruel, viene dato amplissimo spazio alla libertà, mentre l'unica legge è la mancanza di leggi. Nell'abbazia vige infatti la regola del "fai ciò che vuoi". Thélème ricorda in questo la terra di Bengodi, di cui si rinvengono tracce in tutto il pensiero medievale ${ }^{11}$. Scrive infatti Rablais:

La loro vita non era governata da leggi, statuti o regole, ma secondo il loro volere e franco arbitrio. Si levavano dal letto quando loro piacesse; bevevano, mangiavano, lavoravano, dormivano quando ne avevano voglia; nessuno li svegliava, nessuno li forzava né a bere, né a mangiare, né a qualsiasi altra cosa ${ }^{12}$.

Nell'abbazia quindi tutti gli abitanti fanno la stessa cosa. Rabelais giustifica l'omologazione della popolazione grazie al concetto di onore:

Così aveva stabilito Gargantua. La loro regola era tutta in un articolo: "Fa ciò che vorrai," poiché gli uomini liberi, ben nati, bene educati, avvezzi a compagnie oneste hanno per natura un istinto e stimolo che chiamano onore, il quale sempre li spinge a opere virtuose e li allontana dal vizio ${ }^{13}$.

10 "Un essere che per natura non appartiene a se stesso ma a un altro, pur essendo uomo, questo è per natura schiavo: e appartiene a un altro chi, pur essendo uomo, è oggetto di proprietà: e oggetto di proprietà è uno strumento ordinato all'azione e separato. [...] Dunque, è evidente che taluni sono per natura liberi, altri schiavi, e che per costoro è giusto essere schiavi." Aristotele, Politica I, 4-5.

11 Egli infatti scrive:"...in Berlinzone, terra de' Baschi, in una contrada che si chiamava Bengodi, nella quale si legano le vigne con le salsicce, e avevasi un'oca a denaio e un papero giunta, ed eravi una montagna tutta di formaggio parmigiano grattugiato, sopra la quale stavan genti che niuna altra cosa facevan che far maccheroni e raviuoli, e cuocergli in brodo di capponi, e poi gli gittavan quindi giù, e chi più ne pigliava più se n'aveva; e ivi presso correva un fiumicel di vernaccia, della migliore che mai si bevve, senza avervi entro gocciol d'acqua." Boccaccio, Decameron, Giornata VIII, novella III.

12 Rabelais, Gargantua e Pantagruele, Libro I, 57.

13 Rabelais, Gargantua e Pantagruele, Libro I, 57. 
L'abbazia è dunque il luogo in cui l'unica legge è fare ciò che si vuole, nella quale si dà per scontato che tutti seguiranno la propria giusta natu$\mathrm{ra}^{14}$. La spinta combinata di onore e emulazione è la molla che stimola i Telemi a comportarsi in modo giusto, continuando le tradizioni e garantendo la vita all'abbazia. Si tratta dunque di una comunità basata sulla buona volontà e sulla razionalità dei cittadini, caratterizzata da un utopismo ottimistico, basato su principi fondanti della cultura mediterranea, quali l'onore e l'emulazione ${ }^{15}$. Come nota Ullrich Langer ${ }^{16}$ "Faictz ce que vouldras" 17 , vale a dire la mancanza di costrizioni, è il primo momento di partecipazione alla vita dell'abbazia; ma per avere un'abbazia ben ordinata e ben funzionante occorre essere convinti che il "fai ciò che vuoi" risulterà in un'uniformità di azione e d'intenti. Occorre quindi presumere che tutti siano animati dagli stessi intenti, vale a dire che in una situazione di assoluta libertà tutti vogliano il bene comune, non necessariamente il proprio. Ciò, come vedremo, è in contraddizione con il dettato di Thomas Hobbes, secondo il quale, in una situazione di assoluta libertà, ognuno desidererà il bene proprio a scapito di quello degli altri.

L'uniformità di azione ed intenti che caratterizza l'abbazia di Thélème è comune a tutte le utopie rinascimentali e nella finzione letteraria riflette l'uscita dallo stato di natura descritto da Thomas Hobbes. Nelle utopie, una volta entrati nel contratto, le persone rinunciano alla libertà assoluta $\mathrm{e}$ si adattano naturalmente alle leggi della comunità: a Thélème regna la libertà assoluta, che paradossalmente agisce da elemento unificatore. Scrive infatti Rablais: "grazie a quella libertà invece erano presi da emulazione di fare tutti ciò che ad uno vedevano piacere. Se alcuno o alcuna diceva: beviamo! tutti bevevano. Se alcuno diceva: giochiamo! tutti giocavano. Se alcuno diceva: andiamo pei campi a divertirci! tutti vi andavano"18. La valenza utopica consiste proprio in questo: nel fatto che tutti siano convinti di ciò che fanno e agiscano nello stesso modo. Non vi sono ribellioni o sedizioni e ciascuno, per spirito di emulazione e senso dell'onore, segue una serie di leggi condivise.

14 Rabelais, Gargantua e Pantagruele, Libro I, 57.

15 Si veda a questo proposito Honour and Shame, a cura di John Peristiany.

16 Langer, Divine and Poetic Freedom in the Renaissance, 191 e passim.

17 "Faictz ce que vouldras' addresses the reader, offering a vantage point or key to the utopian abbey" Langer, Divine and Poetic Freedom in the Renaissance, 191.

18 Rabelais, Gargantua e Pantagruele, Libro I, 57. 


\section{L'Utopia di Thomas More}

Le utopie scritte da Thomas More, da Campanella, da Anton Francesco Doni e da Francesco Patrizi, per citarne solo alcune, esprimono il desiderio di una società stabile e pacifica ed un modello teso a migliorare quella in cui gli stessi autori vivevano. I concetti di società giusta e libertà possono essere contradditori tra loro, in quanto la giustizia di una società ideale implica una rigidità del sistema che invariabilmente limita la libertà dell'individuo. Ma come è possibile che gli utopisti considerassero migliore un mondo che prevedeva la limitazione sistematica delle libertà personali?

Nell'Utopia di Thomas More la libertà personale viene sacrificata in vario modo. Agli utopiani è fatto divieto di viaggiare liberamente nell'isola; per farlo occorre chiedere il permesso ai magistrati, "ut inops esse nemo aut mendicus possit" commenta Itlodeo: "affinché non vi possano essere né mendicanti né poveri.” In Utopia infatti ogni viandante riceve ospitalità nelle case altrui per tre giorni, dopodiché deve mettersi a lavorare come gli altri. La regola dei tre giorni si potrebbe prestare a molti abusi, ma per non dare la possibilità ai vagabondi di andare di casa in casa ogni tre giorni la mobilità nell'isola viene limitata.

Da un punto di vista pratico, tale regola va collegata all'orrore per il vagabondaggio che caratterizzava la società tardo-medievale. Tale sentimento era ampiamente documentato a livello legislativo, ma per quale motivo? José Antonio Maravall suggerisce che la mobilità territoriale intesa come viaggio e trasferimento era il presupposto di altri tipi di movimento, tra cui la mobilità sociale e la mobilità professionale; essa presupponeva anche l'accesso a professioni diverse e più remunerative. D'altra parte la mobilità geografica si trasformava spesso in una forma di vagabondaggio. Maravall sostiene anche che dalla fine del medioevo il vagabondaggio fu dichiarato fuorilegge nelle città di Castiglia, Inghilterra e Francia. Il divieto di viaggiare in Utopia dev'essere legato ad un timore di questo genere, che corrispondeva alla necessità di una società ben ordinata.

Quindi se da un lato l'utopista si preoccupa che gli abitanti non abbiano a soffrire la fame durante il viaggio, dall'altro, impedendo che le persone si spostino liberamente nel territorio, cerca di evitare che i viandanti diventino vagabondi professionisti. Ciò a sua volta limita la mobilità e l'ascesa sociale, ma si tratta di un piccolo sacrificio in favore del buon funzionamento della società 19 .

19 Maravall, "From the Renaissance to the Baroque," 16-17. A p. 16 Maravall cita anche Leon Battista Alberti, che in Momus, o del Principe, "maintains that those 
Un'altra restrizione presente in Utopia riguarda la religione. In Utopia è ammessa una notevole libertà di culto, specialmente a livello di religiosità interiore. Thomas More scrive infatti che "fra le loro regole più antiche elencano questa: che nessuno possa venire perseguitato per la propria religione" 20 . In quanto "fin dal principio infatti Utopo [...] stabilì che ciascuno era libero di professare la religione che più gli piacesse..."21. Allo scopo di garantire la pace nella comunità le dispute religiose erano vietate: "Chi disputa su questi argomenti con arroganza è punito con l'esilio e la schiavitù"22.

Nonostante il suo destino di polemista contro i luterani e nonostante le vicende biografiche che lo portarono alla decapitazione per la sua strenua difesa della fede cattolica, in questo particolare momento della sua vita Thomas More avvertiva come perniciose le controversie religiose e come salutare un assetto sociale nel quale varie forme di culto fossero egualmente tollerate.

Ogni cittadino era quindi libero di credere qualsiasi cosa, ma vi era una proibizione fondamentale, quella sull'ateismo. In Utopia l'ateismo e la dottrina della mortalità dell'anima erano vietati in modo severo. Si prescriveva infatti "con decreto rigoroso e solenne, che nessuno degradasse la dignità della natura umana al punto di giungere a credere che l'anima perisca col corpo, o che il mondo si regga a caso, senza intervento della provvidenza" 23 .

Si può in questo caso pensare a una semplice deroga alla completa libertà di culto nell' Utopia? Nella discussione sulla religione degli utopiani, More scrive che "Dio vuol essere venerato in modo vario e multiforme"24. In altre parole, la varietà del culto non era in discussione, e per questa vi era piena libertà, ma la mancanza di un culto risultava problematica per More, non solo a livello personale. Forse egli pensava che una negazione di Dio avrebbe potuto mettere in crisi la moralità della società utopiana; si tratta di un timore antico, secondo il quale se Dio è identificato come il summum bonum, la sua negazione non poteva che portare al summum malum. Si può quindi ipotizzare che, in un'ottica di moderazione politica, Thomas More ritenesse che la libertà di culto accordata agli utopiani non

who seek to change their place do not possess social worth or value." Alberti, Momus, 73 e 230 (citato in Maravall, nota a p. 152).

20 More, Utopia, 286.

21 More, Utopia, 286- 287.

22 More, Utopia, 286-287.

23 More, Utopia, 289.

24 More, Utopia, 287. 
dovesse spingersi fino alla negazione di Dio. I vari limiti posti alla libertà individuale nell' Utopia di Thomas More avevano quindi a che vedere con la necessità del buon governo e dell'armonioso funzionamento della città.

\section{L'utopia distopica di Mambrino Roseo}

Ben altre restrizioni della libertà individuale si ritrovano nelle utopie italiane del Cinquecento e del Seicento. Non tutte queste utopie furono scritte con Thomas More come modello. La prima utopia letteraria degna di questo nome è quella proposta da Mambrino Roseo, a sua volta ispirata da un trattato spagnolo di Antonio de Guevara, medico di Santander nelle Asturie, che scrisse un celebre speculum principis intitolato Reloj de princi$p^{25}$. Il libro è noto come Reloj de principes o Libro áureo del emperador Marco Aurelio, in quanto contiene anche alcune lettere attribuite a Marco Aurelio. L'influenza intellettuale e politica di Antonio de Guevara si fece sentire sia durante il regno di Carlo $\mathrm{V}$ che durante quello del suo successore Filippo II. Guevara scrisse anche un libro sulla vita in campagna intitolato Alabanza de aldea y menosprecio de corte. La sua opera ebbe una certa influenza anche in Italia, in particolare nelle aree soggette alla dominazione spagnola; Mambrino Roseo ne trasse ispirazione negli anni '70 del sedicesimo secolo, soprattutto nella parte in cui si narra di una società utopica estinta, che Alessandro Magno avrebbe visitato di persona e di cui avrebbe conosciuto gli abitanti.

È probabile che l'origine di questa storia sia mediorientale. Sebbene non ve ne sia riscontro nell'anonimo Romanzo di Alessandro, scritto in greco e tramandato per tutto il medioevo, l'incontro di Alessandro con personaggi fantastici, giardini edenici e comunità di saggi e filosofi sono ampiamente descritti nelle storie su Alessandro di tradizione islamica, forse per il tramite della Spagna, da cui proviene infatti l'ispirazione di Guevara. Un incontro di Alessandro con una comunità di filosofi è presente, ad esempio, nella vita di Alessandro dello scrittore persiano Nez m (1141-1209)26.

In Mambrino Roseo, la storia dell'incontro di Alessandro con un esponente di una comunità apparentemente utopistica dei Garamanti è l'occasione per illustrare la vita di questo remoto e dimenticato popolo e delle loro abitudini. Essi conducevano una vita spartana e rigidamente strutturata, parsimoniosa e frugale, ossessionata da una logica della scarsità che tra

25 de Guevara, Reloj de Príncipes (1529).

26 Nez mi-ye Ganjav, Il romanzo Iskandarnama (Il racconto di Alessandro) citato in Casari, Alessandro e Utopia nei romanzi persiani medievali, 30. 
loro era assurta a principio universale.

Tra i Garamanti infatti tutto è limitato e rigidamente regolato, primo fra tutti il numero delle leggi. Sono infatti ammesse solo sette leggi (numero cabalistico) e nessuna legge futura può modificare tale regola: "sette e non più sono le nostre leggi, le quali contengono tutta la virtù e tutta la resistenza al vizio. Per la prima è ordinato, che niuno de' posteri faccia altra legge di quelle, che noi gli lasciamo e che a noi sono lasciate" 27.

Motivo ricorrente nelle utopie rinascimentali, l'insoddisfazione verso il sistema legale vigente portava gli utopisti a voler restringere e semplificare il numero delle leggi. Le sette leggi costituiscono una restrizione iperbolica del dettato legislativo, e seguendo il dettato di Guevara, rappresentano il distillato della sapienza Garamante. Come dice "il vecchio Garamanta" sono destinati a perdurare nei secoli.

Come funziona la società Garamante? Lungi dal contemplare la libertà religiosa, nella comunità dei Garamanti solo due divinità possono essere adorate. "che niuno adori più di due Iddii, uno per la vita e uno per la morte" 28 . Il resto della legislazione garamante è caratterizzato da simili restrizioni quantitative. Nella Repubblica dei Garamanti gli abitanti attuano un controllo strenuo e crudele sulle nascite e sulle morti. La loro quarta legge infatti dice: "che niuna donna stia più con suo marito, che quando gli avrà partorito tre figliuoli [...] e se quella donna partorisce altri figliuoli debba essere al cospetto di lei agli Iddii sacrificato"29. Tre è il numero massimo di figli che ad una coppia è dato avere e con una pratica che ricorda l'esposizione dei bambini malati nella città di Sparta, i figli oltre il terzo sono esposti e lasciati morire sotto gli occhi delle madri. Come si vedrà in seguito, a una stretta regolazione delle nascite corrisponde un'altrettanto stretta regolazione delle morti.

Lo stesso spasmodico controllo sulle nascite viene attuato sulle morti. Scrivono infatti "che niuna donna possa vivere più di quaranta anni e l'uomo cinquanta; e se a quella età saran pervenuti, non possa passarli e siano agli Iddii sacrificati" ${ }^{30}$. Alle persone quindi non è dato di morire di morte naturale, e anche se si trattava di età avanzate per quei tempi, le limitazioni alle libertà fondamentali, quali la vita, la riproduzione e il culto lo ren-

\footnotetext{
27 Roseo, "Elogio dei Garamanti," 54-55.

28 Roseo, "Elogio dei Garamanti," 54-55.

29 Roseo, "Elogio dei Garamanti," 54-55.

30 Roseo, "Elogio dei Garamanti," 54-55.
} 
dono un luogo estremamente inospitale.

Il mondo dei Garamanti è quindi al tempo stesso profondamente egalitario e profondamente ingiusto, date le terribili restrizioni imposte alla libertà personale; essi portano alle estreme conseguenze la logica della scarsità presente nelle prime utopie, nelle quali la mancanza di mezzi materiali implica dei limiti allo sviluppo della società. Tutte le antiche società utopistiche dovettero venire a patti con la mancanza di mezzi e di risorse, la cui ridistribuzione equa e razionale era motivo di orgoglio e di buon ordine morale nella comunità.

Società immaginarie come quella di Roseo (e in parte di Campanella e di Thomas More) tendevano a risolvere il problema della scarsità di mezzi e risorse grazie alla moderazione e alla ridistribuzione di ciò che era disponibile. A fronte di risorse scarse o insufficienti, gli scrittori di utopia non tendono a riproporre un'età dell'oro (o un paese di Cuccagna) nel quale le risorse siano infinite, ma una società che funzioni a partire dalla limitazione dei bisogni. Ciò avviene in modo eclatante in Roseo, il quale propone una drastica limitazione delle nascite e della durata della vita umana per sopperire alla scarsità delle risorse, cosa che diventa anche espressione di una inflessibile dirittura morale. In questo regime, che non esiteremmo a considerare come orrendamente totalitario, si avvera l'osservazione di Hitler secondo cui "lo stato totalitario deve ignorare la differenza tra la legge e l'etica"31. In un regime come quello dei Garamanti, è ovvio che legge ed etica hanno confini sfumati e che l'etica viene trasformata in legge. Con questo esempio di proto-totalitarismo, e con quella che di fatto è la prima distopia della storia del pensiero utopistico, si apre la grande stagione delle utopie italiane del Rinascimento.

\section{La libertà nella Città felice di Francesco Patrizi da Cherso}

Alternative classiche a questo schema di estrema scarsità comprendono il già citato Paese di Bengodi, nel quale le risorse sono infinite e dove occorre preoccuparsi di consumare il più efficacemente possibile il cibo che la natura produce in abbondanza; vi sono inoltre alcune rappresentazioni dell'età dell'oro, nelle quali per definizione il problema della scarsità di risorse non ha mai bisogno di venire affrontato 32 .

$\overline{321}$ La frase di Hitler è ricordata in Arendt, Origini del totalitarismo, 430.

32 In questo senso, il modello di sviluppo infinito prevalente invece nella cultura post-industriale, fondato su un uso sempre più efficiente della tecnologia, risale invece al positivismo ma presenta alcune caratteristiche teoriche più simili al paese di cuccagna e all'età dell'oro che all'utopia. Le conseguenze dell'ideologia 
Le altre utopie rinascimentali italiane sono molto meno limitanti. Nella Città felice di Francesco Patrizi infatti si considera una certa quantità di risorse come prerequisito alla vita della città: "Abbia dunque da mangiare e da bere la città se desidera vivere ed esser beata"33, e menziona chiaramente la necessità di un senso di abbondanza necessario a nutrire senza impedimento tutto la città ${ }^{34}$.

La limitazione delle libertà personali, che pure è presente, non nasce quindi dalla necessità di gestire la scarsità delle risorse di un mondo contadino e pre-industriale ma emerge a partire da una struttura filosofica basata sulla Repubblica di Platone e sulla Politica Aristotele. Francesco Patrizi da Cherso scrisse la sua Città felice a vent'anni, dopo aver girato il Mediterraneo lavorando come mozzo sulle galere dello zio. Il risultato è un'utopia di sapore mediterraneo, che si comprende meglio se la si legge avendo in mente e negli occhi la geografia delle isole dalmate. Scrive infatti Patrizi:

sia [la città] in parte edificata sopra colle rilevato, perché sia più esposto all'aure, e, per non aspettare nel medesimo luogo il freddo della vernata che in tali luoghi suole essere più fiero, sia ancora in parte posta nel piano, dove la freddura non può avere così gran forza; ed uno cotal sito non solamente serve alla detta commodità, ma e alla vaghezza della veduta, e alla fortezza ancora della città; e per questo si loda a' tempi nostri Verona ed a' passati Atene ${ }^{35}$.

Se si guarda alla pianta della città di Cherso, così come alla pianta di molte città costali mediterranee, si vede come esse riflettano esattamente questo schema.

La vocazione filosofica di Patrizi lo portò, in età più matura, a divenire professore di filosofia a Ferrara ${ }^{36}$. La profonda conoscenza e ammirazio-

positivista sui destini della terra vengono al momento ridiscusse dal punto di vista di una rinnovata riflessione sull'universale scarsità di risorse (o sulla loro finitezza) sulla terra. Tale riflessione presenta quindi degli aspetti utopici, basati sulla necessità di consumare meno risorse possibili e apre a scenari distopici, secondo la persuasione che uno sviluppo infinito (o anche uno "sviluppo sostenibile") sia incompatibile con ciò che la terra è in grado di offrire.

33 Patrizi, La città felice, 92.

34 Patrizi, La città felice, 92.

35 Patrizi, La città felice, 95.

36 Si veda tra gli altri Vasoli, "La critica di Francesco Patrizi alla dottrina aristotelica degli elementi," 93-106. 
ne per Platone e Aristotele emergono già nella Città felice, il cui originale disegno platonico delle tre classi sociali (lavoratori, guerrieri e governanti) è raddoppiato secondo lo schema aristotelico, in modo da riflettere la maggiore complessità della vita urbana del Rinascimento ${ }^{37}$. La Città Felice è composta infatti di sei classi sociali: contadini, artigiani, mercanti, guerrieri, magistrati e clero: "alla costituzione di una città beata, sei maniere d'uomini si ricerchino" 38 . Secondo il dettato platonico, ogni cittadino riveste solamente una funzione, né può risalire l'ordine sociale. Il ruolo dei cittadini è infatti limitato alla loro funzione sociale, che viene decisa al momento della nascita. Si nota anche come la differenziazione della società in classi privilegiate e classi lavoratrici si possa far risalire alla divisione aristotelica tra uomini liberi e schiavi 39 .

Alla base del buon funzionamento della Città Felice vi è dunque una fondamentale ineguaglianza sociale, garantita da un collegio di ottimati che presiedono ai destini della città. Essi sono eletti a turno, sul modello di Venezia. Ai cittadini delle tre classi inferiori non è dato di accedere alle acque celesti, perché non è dato loro fare esperienza della felicità. Patrizi segue qui il dettato aristotelico, per il quale la virtù si addice ad una vita di otium, nella quale non sia necessario affaticarsi per vivere. "anzi tutta la vita loro spendono in affaticarsi, per far vivere e sé e gli altri; per i quali impedimenti non possono acquistarsi l'operazione e gli abiti delle virtù, le quali sono quell'ultimo passo, che ci fanno alla beatitudine arrivare" 40 . Gli uomi-

37 È noto che nella Repubblica di Platone la società è costituita di tre classi sociali: contadini, governanti e guerrieri. È opinione diffusa che La città felice sia infusa di platonismo e neoplatonismo e che Patrizi abbia raddoppiato il numero delle classi sociali preservando la struttura della Repubblica di Platone. Sul (neo)platonismo di Francesco Patrizi si veda Muccillo, "Marsilio Ficino e Francesco Patrizi da Cherso" e "Aristotelismo, platonismo ed ermetismo ne "La città felice»." Mislav Kukoč fa invece notare come nella Politica di Aristotele la società sia composta di sei classi: contadini, artigiani, guerrieri, ricchi, sacerdoti e giudici e nella Città felice la struttura sociale sia molto simile: contadini, artigiani, mercanti, soldati, magistrati e sacerdoti. (Kukoč "Petrič's Socio-Philosophical Thought Between Realism and Utopianism," 414-415).

38 Patrizi, La città felice, 92.

39 Si vedano a questo proposito le riflessioni di Kukoč in "Petrič's SocioPhilosophical Thought Between Realism and Utopianism," 415, che cita Aristotele Politica, 1329a26 e 1330a25-30.

40 "Queste sei predette maniere d'uomini, che di compagnia si misero a sí faticoso camino, beeranno elleno tutte dell'acque sopracelesti? Certo tutte quelle 
ni che devono affaticarsi per vivere non possono essere virtuosi e quindi non possono raggiungere la felicità.

L'aspetto più caratteristico dell'utopia di Patrizi è la suggestiva metafora delle acque celesti, costruita con elementi plotiniani, evangelici e francescani ${ }^{41}$. Se il raggiungimento delle acque celesti è permesso solamente alle classi superiori, esse abbisognano di quelle inferiori per il buon funzionamento della società. Ai cittadini delle classi inferiori non è data la possibilità di accedere alle classi superiori né di attingere alle acque celesti. La loro vita è appiattita sulla loro funzione poiché il loro unico ruolo nella città è il meccanico funzionamento della stessa. La sopravvivenza delle classi superiori è invece finalizzata al raggiungimento dello stato di grazia, mentre la perpetuazione delle classi inferiori è finalizzata all'esistenza della città.

La mancanza di libertà nella Città Felice risulta evidente dalle condizioni di vita delle classi inferiori; il fatto che non a tutti sia permesso di ottenere lo stato di grazia simboleggiato delle acque celesti attesta infatti della limitazione alla libertà individuale. Si obietterà che almeno tre delle classi sociali sono di fatto libere e probabilmente felici. Vale però la pena ricordare qui il dettato di John Rawls, il quale, per giudicare la desiderabilità di una società immaginaria o reale propone il seguente criterio: una società è giusta se chiunque desidererebbe viverci senza tener conto (o conoscere) la posizione sociale che occuperà nella stessa ${ }^{42}$. Secondo questo criterio, la Città felice è ingiusta, perché è desiderabile vivere nelle tre classi superiori, ma non necessariamente nelle tre inferiori.

Nella Città felice di Francesco Patrizi quindi, la mancanza di libertà per le classi inferiori deriva non da una necessità intrinseca di ridistribuire le poche risorse disponibili, ma da una necessità filosofica, di distinguere le classi sociali che sono naturalmente virtuose da quelle non virtuose. Da ciò ne consegue una differenziazione sociale che limita fortemente la libertà delle classi considerate non virtuose. A differenza dell'utopia di More e di quella di Roseo quindi, la mancanza di libertà ha qui in primis origini filosofiche, e solo successivamente motivazioni pratiche.

saranno dell'acque saziate e felici, a cui converrà la diffinizione della felicità." Francesco Patrizi, La città felice, 92.

41 Piaia "Entre le mysticisme Platonicien et la "Philosophie des fleuves," passim e in particolare le pagine 384 e 385. Perissinotto "L'utopia mediterranea di Francesco Patrizi da Cherso," 151-160.

42 Questo criterio è menzionato da Levitas, The Concept of Utopia, 184. 


\section{Il mondo savio e pazzo di Anton Francesco Doni}

Nell'utopia di Anton Francesco Doni intitolata Il Mondo Savio e Pazzo $0^{43}$ il miglioramento delle condizioni economiche avviene tramite l'abolizione della proprietà privata e del denaro. La società è strettamente comunistica perché va nella direzione di una rigidissima distribuzione dei beni, grazie alla quale, sostiene Doni, ciascuno può ottenere tutto ciò che desidera: "Per che cosa s'aveva egli da litigare? Tutto era comune, e i contadini vestivano come quei di città; perché ciascun portava già il frutto della sua fatica, e pigliava ciò che gli faceva bisogno" 44 . Si tratta ovviamente di una nozione aporetica, nel senso che dall'eguaglianza di diritti e doveri non segue necessariamente l'estinzione di ogni desiderio. Di quest'aporia Doni non si dà pensiero perché la sua preoccupazione si situa altrove. Le restrizioni che egli propone infatti sono legate principalmente alla vita affettiva e alla sfera dei sentimenti, che egli considera perniciosi sia a livello individuale che a livello sociale. Egli vede come particolarmente dannoso il tormento d'amore, problema sentito e affrontato in vari modi a partire da Ovidio e rielaborato sia nell'antichità classica che per tutto il medioevo.

Secondo Doni l'amore si forma per emulazione dal contatto con il seno della madre; causa dei mali del mondo, esso va eliminato dalla società fin dalla nascita degli individui. L'unico modo di eliminarlo è la separazione dei neonati dal seno della madre non appena essi vengono alla luce. "Una strada o due di donne, e andava a comune la cosa" 45 commenta Doni, che riduce la riproduzione a un mestiere da destinare, come gli altri mestieri, a una o due strade della città.

"Onde, continua Doni, non si sapeva mai di chi uno fosse figliolo, e la cosa andava pari" 46 . Essendo i bambini nati tutti nello stesso luogo, la teoria è che si sarebbero confusi tra loro, e che nessuna madre avrebbe saputo a quale dei bambini allevati dalla comunità ella aveva dato la vita. Tale prospettiva è salutata con grande interesse da uno dei due protagonisti del dialogo, che commenta: "benedetto sia questo paese! ... onde non si doveva mai piangere" 47 . L'eliminazione del sentimento d'amore e del dolore porta alla dissoluzione della famiglia e permette anche di selezionare più efficacemente i cittadini più sani con la pratica (già messa in atto nell'antica

\footnotetext{
43 Scritta nel 1552, pubblicata come Doni, Il mondo savio e pazzo (1552)

44 Doni, Il mondo savio e pazzo, 74.

45 Doni, Il mondo savio e pazzo, 73.

46 Doni, Il mondo savio e pazzo, 73.

47 Doni, Il mondo savio e pazzo, 73.
} 
Sparta) di "esporre" i bambini malati. Si può ipotizzare che questa utopia così radicale sia, al pari di quella di Thomas More, almeno in parte ironica nei confronti della società che descrive: è possibile infatti che si ironizzi verso quei modelli sociali anche ideali, come quella di Patrizi per esempio, in cui si privilegiavano l'amore, l'armonia e i sentimenti.

L'eliminazione dei sentimenti è un progetto chiaramente irrealizzabile. Non è possibile eliminare i sentimenti dalla vita umana senza soffrire di tutta una serie di ripercussioni che andrebbero a ledere la libertà personale molto più di quanto non lo faccia la schiavitù d'amore che Doni individua come responsabile dei mali del mondo. Ma le raccomandazioni del Doni possono essere interpretate nel senso di un accrescimento della libertà personale che affranca le persone dal giogo della famiglia. Ma quando si guarda alle possibili conseguenze di tale assetto sociale ci si rende conto che $\mathrm{i}$ danni sarebbero tali e tanti da oscurare i benefici di tale libertà.

\section{Onnipotenza e libertà in Ludovico Agostini}

Sarà Ludovico Agostini nei Dialoghi dell'infinito, scritti tra il 1575 e il 1580 a dare alle stampe la prima utopia post-tridentina. Stato fortemente militarizzato (secondo il modello della polis classica), la società descritta da Agostini è divisa, platonicamente, in tre classi sociali. In essa si prevede sia l'istituzione della famiglia sia una limitata proprietà privata; ma non è permessa nessuna deviazione dal culto ufficiale, cosa che esclude musulmani, ebrei e cristiani "scismatici", vale a dire non cattolici.

La vita quotidiana degli abitanti è rigidamente regolata, così da evitare che essi si diano all'ozio e ad altri vizi. Per certi versi la società è regolata come un convento: "ordineremo che tutto il popolo due volte la settimana abbia a stare di un sol pasto contenuto" 48 . Se il digiuno si rende necessario, dice Agostini, per "evacuare la superfluità degli umori”"49, la confessione e la preghiera ad ore specifiche (dopo pranzo per esempio) sono necessarie per conservare "la sanità dell'anima" 50 , e per combattere le tentazioni. Aspre sanzioni sono comminate a chi non ottempera a queste regole. In quest'utopia gli abitanti vengono particolarmente infantilizzati; la longa manus dell'autore arriva fino alla scelta del tipo di materasso da mettere sul letto (semplice e non di piume) al numero di ore in cui ciascuno può dormire (sette).

48 Agostini, La repubblica immaginaria, 141.

49 Agostini, La repubblica immaginaria, 141.

50 Agostini, La repubblica immaginaria, 140. 
Luigi Firpo, nell'introduzione all'edizione del 1957 della Repubblica immaginaria, insiste sul valore storico di questa utopia, nata subito dopo la controriforma e concepita per limitare gli effetti di una società viziosa e avida. Sostiene inoltre Firpo che il "mondo ideale, severo, mortificato dalle rinunce" di Agostini era supportato dalla sua fiducia nell'educazione ${ }^{51}$. Alla base della Repubblica immaginaria di Agostini vi sarebbe secondo Firpo una fondamentale tensione, mai risolta, tra il mondo controriformista, con la sua preponderanza del principio di autorità, e un "esile razionalismo umanistico" 52 che per bocca di Finito, uno dei protagonisti, tenta di sopravvivere al nuovo clima politico e religioso controriformista.

Seppur storicamente determinate, come sostiene Firpo, le istanze oppressive nella Repubblica immaginaria ne fanno un'utopia particolarmente difficile e indesiderabile; tale società, basata su un elaborato rituale militare e religioso, assomiglia più a un convento militarizzato che a un luogo in cui vivere secondo giustizia. Secondo i dettami dell'ideologia controriformista, nella Repubblica di Agostini il divino è onnipotente, onnipresente e pervade ogni aspetto della vita degli abitanti, al punto che viene usato come mezzo di controllo sociale.

Alla divinità onnipotente Agostini infatti accorda massima libertà e massima presenza nella vita degli uomini. Il discorso dell'assoluta onnipotenza di Dio si ripresenta, secolo dopo secolo, nella discussione filosofica e teologica cristiana, originando infinite variazioni della visione del divino. Si va dall'occasionalismo di Al-Ghazali, secondo cui l'unica causa efficiente è Dio ed è quindi Egli stesso a generare direttamente tutti gli effetti del mondo, a Cartesio, il quale conferisce sia all'uomo che a Dio completa libertà. Rimane il problema di conciliare l'assoluta libertà di Dio, la sua assoluta benevolenza, con la presenza del male del mondo, e nello stesso tempo conciliare con questi due concetti l'idea della libertà dell'uomo ${ }^{53}$.

Vi è una teoria secondo la quale l'assoluta onnipotenza e libertà di Dio abbiano dato inizio all'epoca moderna e ad una visione dell'uomo svincolata dalla divinità. Infatti se Dio è infinitamente libero, allora Egli diviene indecifrabile e finisce per nascondersi alla ragione umana. In altre parole l'era del deus omnipotens dà origine al deus absconditus, il quale porta alla

51 Firpo, Introduzione a Agostini, La repubblica immaginaria, 12.

52 Firpo, Introduzione a Agostini, La repubblica immaginaria, 12.

53 Marlin, Cartesian Freedom and the Problem of the Mesland Letters, 151-172. Il saggio presenta una discussione abbastanza completa di questi problemi, nonché un'adeguata silloge della bibliografia in francese sul concetto di libertà in Cartesio. 
graduale scomparsa di Dio, nel senso che "un Dio nascosto è come se fosse morto" 54 .

Nel suo totalitarismo, Il mondo savio e pazzo di Anton Francesco Doni vede la divinità come immensamente libera e onnipresente e crea un'autorità terrena che ottemperi al Suo disegno sulla terra. Quest'utopia è narrata come se fosse un sogno, dimostrando quanto tenue sia il legame con il mondo reale e come l'autore non si faccia limitare dala realtà nella descrizione. Nonostante il regime fortemente totalitario e militarizzato, il tono dell'opera vuole essere umoristico, il che fa pensare che si tratti, almeno in parte, di un intervento satirico, ove la parte ludica e destruens sia volutamente più incisiva della pars construens.

\section{La libertà dei Solari}

Seppure espresse diversamente e dirette verso un'altra sfera della vita umana, anche nella Città del sole di Campanella esistono infinite limitazioni della libertà personale 55 . I Solari erano dediti a complesse pratiche eugeniche, che Campanella potrebbe aver osservato nell'allevamento dei cavalli56. La riproduzione era strettamente regolata dal governo centrale sulla base di criteri astrologici. Secondo Campanella

[uomini e donne] dormeno in due celle, sparti fin a quell'ora che si han da congiungere, ed allora va la maestra, ed apre l'uscio dell'una e l'altra cella. Questa ora è determinata dall'Astrologo e Medico; e si forzan sempre di pigliar tempo, che Mercurio e Venere siano orientali dal Sole in casa benigna e che sian mirati da Giove di buono aspetto e da Saturno e

54 Si veda Ulrich Langer che cita Hans Blumemberg: "A hidden god is pragmatically as good as dead." Langer, Divine and Poetic Freedom in the Renaissance, 8.

55 Anche la Città del Sole prende le mosse, seppur lontane, dalle gesta di Alessandro. Secondo l'anonimo del Romanzo di Alessandro, in una lettera alla sua augusta madre il condottiero scriveva così: "poi ripartimmo e giungemmo fino al fiume Atlante. [...] Oltre quel luogo c'era una terra [...] ci imbarcammo e arrivammo alla città del Sole che aveva un perimetro di centoventi stadi [...], c'erano quattordici torri costruite in oro e diamanti. Le mura erano di pietra indiana. Al centro c'era un altare fatto di oro e diamanti, con sessanta gradini. Sopra c'era un carro con cavalli d'oro e di smeraldi. Non era facile vedere tutto questo per via della nebbia. Il sacerdote del sole era un Etiope [...] poi trovammo il buio. Allora decisi di sacrificare ancora al sole." Anonimo, Romanzo di Alessandro. 154. Si veda anche supra, nota 26.

56 Headley, Tommaso Campanella and the Transformation of the World, 44. 
Marte... E per lo più vogliono Vergine in ascendente ${ }^{57}$.

Le donne erano in comune, ma di fatto non era permesso agli uomini di unirsi e procreare con qualsiasi donna, solo con alcune particolari donne scelte all'interno della comunità. Si noti come la libertà delle donne di procreare o meno non venisse nemmeno presa in considerazione. Anche la scelta del compagno era determinata da criteri generali, dei quali l'amore o le scelte individuali non facevano parte. Criteri utili erano il miglioramento della specie e l'attrazione degli opposti. "Talché si guarda bene, e si donano questi [uomini sapienti, che "han debole lo spirito animale"] a donne vive, gagliarde e belle; e gli uomini fantastichi e capricciosi a donne grasse, temperate, di costumi blandi” ${ }^{58}$. Ma se due membri della comunità eccellevano in qualcosa, ad esempio erano i più belli o i più forti essi dovevano accoppiarsi e riprodursi tra loro. In tutti gli altri casi valeva la legge degli opposti: uomini nervosi con donne calme, intellettuali con donne energiche, ecc.59.

Anche il momento e la frequenza dei rapporti sessuali erano scelti accuratamente in base a criteri astrologici e autorizzati dal governo centrale: "sendo ben lavati, si donano al coito ogni tre sere," dormendo in celle separate da una porta che al momento più propizio veniva aperta da funzionari addetti. A quel punto la coppia era in grado di consumare l'atto sessuale. Campanella aggiunge che "sendo generati della medesima costellazione, li contemporanei sono di virtù consimili e di fattezze e di costumi. E questa è concordia stabile nella Repubblica e s'amano grandemente ed aiutano l'un l'altro." Campanella riteneva dunque che la determinazione astrologica del concepimento facesse sì che tutti i nati sotto una certa stella avessero caratteri simili e che per questo stesso motivo si potessero governare più facilmente ${ }^{60}$.

In un certo senso, tutte queste restrizioni alla riproduzione libera tendevano ad allontanare la variabile "intrattabile" dell'amore dal processo

57 Campanella, La città del Sole, 50.

58 Campanella, La città del Sole, 50.

59 “e li sapienti [...] per la molta speculazione, hanno debole lo spirito animale e non trasfondendo il valor della testa, perché pensano sempre a qualche cosa; onde trista razza fanno." Campanella, La città del sole, 47.

60 Campanella, La città del Sole, 48. Concetto ricorrente questo, che viene ripreso anche in epoca contemporanea. Aldous Huxley in Brave New World, scrivew che tutti gli abitanti sono concepiti in provetta da tecnici i quali clonano chimicamente centinaia di "gemelli" facilmente governabili perché chimicamente modificati tutti alla stessa maniera. 
riproduttivo. Ciò era utile a Campanella per garantire che la comunità si riproducesse oltre la prima generazione. Al di fuori di tale schema riproduttivo ognuno era libero di innamorarsi e di unirsi alle altre persone come volesse. La politica sessuale di Campanella è del tutto mirata al mantenimento delle generazioni future nella Città del Sole. Al di fuori di questo schema la famiglia non è necessaria e le persone possono amarsi come credono.

Sia nel caso dei Dialoghi dell'infinito che nella Città del Sole gli autori immaginano una comunità composta di filosofi, formatasi spontaneamente e senza costrizioni. Le regole e costrizioni hanno per lo più a che vedere con il modo in cui la comunità viene perpetuata nel tempo ${ }^{61}$. Il discorso di Campanella è teorico-filosofico ma anche eminentemente pratico. Campanella aspirò, prima ancora di comporre La Città del Sole, alla monarchia universale, con tutta probabilità identificata nella monarchia spagnola; la sua utopia va interpretata dunque come un distillato della scienza campanelliana su questioni morali, demografiche e politiche ${ }^{62}$.

\section{Lodovico Zuccolo e la libertà}

Un discorso radicalmente differente è quello che Lodovico Zuccolo propone nel dialogo intitolato Il Belluzzi e sottotitolato La città felice. In esso Zuccolo vi intesse le lodi di San Marino, borgo famoso per la libertà dei cittadini e l'indipendenza dai potentati vicini. L'autore lo descrive come regno della libertà, nel quale ogni cittadino è autonomo e può fare ciò che vuole,cosa che funziona da cardine, principio unificatore e panacea. "Dove regna la libertà senza licenza, non si pongono taglie e tasse senza necessità, non si dànno gli onori per danari o per favore, non si astringe nessuno ai carichi contra sua voglia" 63 Si tratta di una repubblica reale e nel contempo idealizzata, presente agli occhi dello scrittore di utopia come mondo già

61 Il problema di far sopravvivere la comunità oltre la prima generazione è fondamentale sia nelle utopie antiche che nelle utopie contemporanee, le cosiddette comunità intenzionali. Per esempio nella comunità di Twin Oaks (www.twinoaks.org) una delle "comuni" ancora funzionanti negli Stati Uniti, gli abitanti normalmente stentano a trattenere i propri figli all'interno della comunità. Pur rimanendo emotivamente legati a Twin Oaks, i figli tendono a scegliere per sé ruoli sociali più tradizionali.

62 Si veda l'illuminante capitolo su Campanella di Friedrich Meinecke in Lidée de la raison d'Etat dans l'histoire des temps modernes, 89-112.

63 Zuccolo, Il Belluzzi, 38. 
esistente e perfettamente compiuto.

Libertà e uguaglianza sono alla base di questa insolita utopia, che scardina tutti i canoni delle narrazioni utopiche precedenti e ne stabilisce di nuovi. Se questi nuovi criteri fossero stati esplorati e sviluppati, avrebbero dato seguito a un filone diverso e nuovo nella letteratura utopistica. Due sono i principî che rendono felice questa città: una è la frugalità del popolo, che Zuccolo interpreta come povertà condivisa. Egli ne fa una specie di apologia, dicendo tra l'altro che: "dove tutti siano assolutamente poveri, ma tuttavia commodi la più parte rispetto alla semplicità del viver nostro [...] non ha potuto introdurvi né la tenacità del proprio, né l'ingordigia di quello d'altrui" 64 . Egli riassume così un'idea già ampiamente sfruttata nelle utopie precedenti, vale a dire che la cupidigia viene allontanata con una combinazione di frugalità e uguaglianza.

Infatti il secondo principio su cui si basa Il Belluzzi è una dignitosa uguaglianza, definita da Zuccolo come "quella mediocrità, nella quale la quiete e la felicità pubblica mettono radici”"65. La scarsità e la libertà degli uffici e la pregevole locazione sono gli altri fattori che, uniti a leggi sagge ed eterne, fanno sì che questa città sia migliore delle altre e non abbia a soffrire di nulla.

L'autore non fornisce molti dettagli e le caratteristiche di tale città sono destinate a rimanere in parte misteriose, anche per una forma di isolamento atto a preservarne la purezza e la virtù: "Qui, scrive Zuccolo, non vengono forastieri a corrompere i nostri costumi, non mercanti ad introdurvi delizie, non banchieri a distruggerci co' cambi... ecc.". La vita isolata sembra un requisito fondamentale di questa città, nella quale, dice Zuccolo, "i nostri vicini istessi non sanno bene a dentro la felicità di questa Repubblica, i lontani né pur la conoscono per nome. Così, oscuri agli altri, viviamo celebri a noi soli" 66 .

Trattandosi di un'utopia che traccia il ritratto di una città esistente, il Belluzzi sarebbe a rigore una laudatio urbis, giustificata anche sulla base di confronti con celebri città del passato, quali Sparta e Atene. È vero che ogni grande città del passato ha creato il proprio panegirico, ma raramente è arrivata a concepire se stessa come utopistica. Pensare che una società sia perfetta e poterci vivere è cosa da visionari o da uomini molto fortunati.

Lodovico Zuccolo è l'autore di un'altra utopia, immaginaria e platonica, ispirata all'utopia di Thomas More e intitolata la Repubblica di

64 Zuccolo, Il Belluzzi, 39.

65 Zuccolo, Il Belluzzi, 38.

66 Zuccolo, Il Belluzzi, 40. 
Evandria ${ }^{67}$. A differenza del Belluzzi, la Repubblica di Evandria s'inserisce nella temperie culturale e politica contoriformista. Le restrizioni alla libertà degli Evandri vanno dalla limitazione all'uso della moneta (già presente in Utopia) all'isolamento dal mondo esterno tramite la restrizione delle visite degli stranieri, presente anche nelle comunità utopistiche del Paraguai, che si ispiravano probabilmente all'utopia di Campanella. In Evandria vigono varie limitazioni allo sviluppo culturale, come la regolazione della circolazione dei libri, regolata dai magistrati e la limitazione delle leggi, già notata nell'utopia di Campanella e nella distopia ante litteram di Mambrino Roseo.

\section{Conclusioni}

La tensione tra libertà dei cittadini e le regole di buon funzionamento dello stato caratterizzano tutte le utopie che abbiamo analizzato in questo saggio. Tale ambiguità si presenta ogniqualvolta si parli di utopia, e può essere compresa solo contestualizzando il discorso utopistico all'interno della filosofia medievale e rinascimentale. Una prima considerazione da fare ha a che vedere con il concetto di volto nascosto di Dio, mutuato dalla teologia nominalista. Come si è visto sopra, sin dal medioevo, più si conferisce onnipotenza a Dio più se ne potenzia la libertà. Un Dio assolutamente libero diviene imperscrutabile, e il suo volto finisce per nascondersi alla visione degli uomini, che avocano a sé (o si riprendono) parte della libertà attribuita a Dio. Il volto nascosto di Dio e la sua infinita libertà fanno sì che l'autorità venga trasposta in parte da Dio agli uomini, con il risultato di una graduale secolarizzazione della società.

Una seconda considerazione è quello del rapporto tra libertà e diritto. La libertà poteva essere dentro o fuori dall'autorità e dalla legge, dentro 0 fuori dal diritto, vale a dire poteva essere regolata all'interno delle strutture giuridiche o considerata assoluta, e perciò stesso al di fuori di esse, come nello stato di natura di Thomas Hobbes. Nelle utopie rinascimentali la libertà era regolata dalle leggi, vale a dire era regolata dal diritto.

D'altra parte, secondo la riflessione del giusnaturalismo, vale a dire del diritto naturale, che sia Campanella che More rispettavano, le libertà erano percepite come anteriori al diritto. In seguito sia Hobbes che Locke avrebbero chiarito la nozione che la libertà era anteriore al diritto e che il diritto doveva intervenire per regolarla.

In particolare, Thomas Hobbes concepì la libertà degli uomini come compatibile con la necessità. Psicologicamente le azioni che compiamo sono 
determinate dai nostri appetiti e gli individui dispongono della libertà di seguirli o meno. Hobbes era determinista, nel senso che pensava che tutte le nostre azioni fossero determinate da appetiti di tipo meccanico - e compatibilista, nel senso che pensava che il suo determinismo fosse compatibile con la libertà personale68. D'altro canto John Locke vedeva alla radice della libertà una sospensione del desiderio; in altre parole, l'uomo era libero quando non era più governato dal desiderio e in grado di fare una scelta basata sull'analisi, vale a dire sulla razionalità, e agire di conseguenza. Dato che la razionalità è uguale per tutti, tale concezione della libertà dà luogo a comportamenti simili in tutta la popolazione che si sia liberata dei desideri69.

Per contro, alla base della riflessione sulla libertà nelle utopie italiane del Rinascimento vi è l'idea che il mondo riposi su una serie di cause pratiche e meccaniche e che l'universo vada concepito all'interno di costruzioni politiche che siano sia ideali (nel senso di utopistiche) che pratiche ${ }^{70}$.

68 Chappell Hobbes and Bramball on Liberty and Necessity, xi. Nell'introduzione, Chappell traccia un'utile distinzione tra compatibilismo, incompatibilismo e determinismo. Per Hobbes, la libertà è compatibile con la necessità (con un universo necessario), nel senso che tutte le azioni umane sono necessitate da una causa. In "Self-determination," Vere Chappell analizza invece il punto di vista opposto, quello di esseri che autodeterminano le proprie azioni, punto di vista proposto, oltre che da John Bramhall (il quale riteneva che alcune azioni non fossero necessitate da una causa antecedente ma fossero frutto del libero arbitrio) anche da John Locke e fa risalire le sue convinzioni, almeno in parte, alle dottrine di San Tommaso d'Aquino. Vere Chappell, "Self-Determination," 141 e passim.

69 "We have the power to suspend the prosecution of this or that desire, as every one daily may Experiment in himself. This seems to me the source of all liberty; in this seems to consist that, which is (I think improperly) cll'd Free will. For during this suspension of any desire, before the will be determined to actions, and the action (which follows that determination) done, we have opportunity to examine, view, and judge, of the good or evil of what we are going to do; and then, upon the Examination, we have judg'd, we have done our duty, all we can, or ought to do, in pursuit of our happiness; and 'tis not a fault, but a perfection of our nature and desire, will, and act according to the last result of a fair Examination. This is so far from being a restraint or a diminution of Freedom. That it is the very improvement and benefit of it: 'tis not much an Abridgement, 'tis the end and use of our Liberty." Locke, An Essay Concerning Human Understanding, 47-48. Si veda anche Chappell, "Locke on the Freedom of the Will."

70 Il realismo delle utopie rinascimentali è stato ampiamente illustrato da EliavFeldon in Realistic Utopias. 
Spesso nelle utopie che abbiamo visto si delinea una precisa forma di iperregolazione, dovuta al fatto che il diritto naturale viene sostituito dal diritto della società. È normale per le società filosofiche, nelle quali le persone entrano spontaneamente, far decadere alcuni diritti naturali in funzione di un miglior funzionamento della società ${ }^{71}$. Una limitazione della libertà si potrebbe quindi facilmente giustificare nel caso di società di tipo volontaristico, come la Città del Sole di Campanella, ma che fare dei membri delle società utopistiche che non sono volontari? Che fare della seconda generazione dei Solari?

Occorre presupporre comunque una forma di contrattualismo, attraverso il quale le società civili si formano al di là della legge naturale. I membri della società che non sono in grado di comprendere la necessità dell'ingresso di tutti gli individui nel consesso civile devono essere obbligati a farne parte, per il loro bene e per il bene comune. Essi di conseguenza perdono la libertà assoluta dello stato di natura, ma acquistano il diritto di far parte di un consesso civile in cui le contraddizioni e le lotte interne sono state risolte. Peraltro nelle utopie non si ritiene che il potere politico possa essere esercitato per motivi personali, né che la volontà dei governanti possa essere distinta dalla volontà del popolo ${ }^{72}$; in nessuna delle utopie fin qui analizzate si ammette di tirannicidio, anzi si cerca in tutti i modi di evitarlo. Esso è un'azione incompatibile con l'utopia (che non è per definizione una tirannide) e con l'idea che i governanti facciano sempre l'interesse dei cittadini.

Nel contesto politico e culturale del Cinquecento, non sembra ravvisarsi un'incompatibilità tra libertà e autorità. Nell'utopia teocratica di Ludovico Agostini il diritto divino è prioritario rispetto a quello umano, quindi la libertà individuale viene in secondo o in terzo ordine, dopo la libertà di Dio e dopo aver assolto alle necessità dello stato. Secondo la cor-

71 Peraltro John Rawls sostiene che ciò non è possibile, e che la libertà ha un ruolo primario e prioritario nella formazione delle società. Egli sostiene infatti che una volta stabilite in modo efficace le libertà fondamentali esse non possano essere sottratte nemmeno in parte, o alienate in cambio per esempio di una migliore condizione economica, con la quale s'intende sia quella della società intera che quella dell'individuo. ("The priority of liberty means that whenever the basic liberties can be effectively established, a lesser and/or unequal liberty cannot be exchanged for an improvement in economic well-being." Rawls, A Theory of Justice, 132).

72 Tale punto di vista sarà poi formulato pienamente da John Locke. Si veda Ashcraft "Locke’s Political Philosophy," 228. 
rente giusnaturalista, lo stato garantisce legalità e legittimità, mentre la libertà è permessa ad un altro livello, quello della coscienza.

Ciò si riflette anche in alcune delle utopie più liberali, come quella di More e Campanella che ammettono la libertà di culto ma non tollerano ateismo e proselitismo. La libertà si configura quindi principalmente come libertà di coscienza. In questa temperie politico-culturale, non vi è incompatibilità tra autorità e libertà. La libertà è compatibile con l'autorità (Locke) e la libertà è fuori dal diritto (Hobbes), che regola tutto, ma non è sentito come una limitazione della libertà. Se Dio è nascosto egli diventa imprevedibile; la faccia nascosta di Dio costringe le persone ad occuparsi di più della realtà che li circonda, per il fatto di non essere in grado di decifrare la libertà di Dio, che diviene una grandezza tanto incommensurabile per la mente umana quanto incomprensibile.

Possiamo presumere che se Dio si nasconde, a un certo punto possa scomparire anche la sua autorità e che essa venga dislocata da Dio alla mente del legislatore utopista. La libertà di Dio deve essere concepita ai margini, ma come parte integrante della riflessione sull'utopia, mentre la libertà dell'uomo viene onorata principalmente nella sua coscienza. Tutto ciò risulta più semplice nei casi di comunità filosofiche di tipo volontaristico, ma per garantire la libertà della seconda generazione, o quella di comunità non filosofiche e di tipo non-volontaristico occorre presupporre che tutti gli utopiani siano sempre d'accordo, senza però sapere in che modo questo accordo avvenga. Oppure occorre attendere che Thomas Hobbes chiarisca i criteri adeguati per giustificare l'entrata nel consesso sociale degli elementi riluttanti: coloro che si oppongono lo fanno per ignoranza; vengano quindi educati e costretti a unirsi al consesso civile.

Nello stato di natura la libertà è assoluta; quando si abbandona lo stato di natura, ci si lascia indietro anche la libertà assoluta per il privilegio di poter vivere in uno stato più giusto ed egalitario. Nel consesso civile la libertà assoluta viene ridotta e limitata, mentre fin dal medioevo e nelle utopie più teocratiche si assolutizza la libertà di Dio relegando quella umana al mondo interiore.

In questo senso, uno degli aspetti dell'utopia che stanno più a cuore ai lettori moderni, quello della libertà politica e dell'autonomia personale non è di grande rilievo, nel senso che la libertà delle utopie rinascimentali era molto lontana dal concetto kantiano di libertà come autonomia ${ }^{73}$. Nel

74 "Che altro dunque, può essere la libertà della volontà, se non l'autonomia, i.e. la proprietà che ha la volontà di essere legge a se stessa?" Kant, Fondazione della metafisica dei costumi, 447. Kosch, Freedom and Reason in Kant, Schelling and Kirkegaard. 
discorso utopistico del Rinascimento si gioca invece un'altra, molto più importante partita: la possibilità di avere una società efficiente, libera dalle pastoie delle disuguaglianze medievali, nella quale si potesse ipotizzare una soluzione dei conflitti sociali.

Oggigiorno la libertà viene da un lato considerata un bene assoluto e dall'altro un bene in costante pericolo, proprio nel momento in cui il suo significato viene ridiscusso a livello politico internazionale ${ }^{74}$. Nelle utopie rinascimentali la libertà non fu mai un bene onnicomprensivo, un presupposto e una priorità, ma un concetto limitato spesso all'interiorità e alle costanti mutazioni delle differenti forme dell'utopia. Alla libertà veniva dato più o meno spazio a seconda della necessità di far funzionare la comunità in modo razionale ed efficiente. Si tratta quindi di un concetto elusivo, proteiforme e dipendente dalle decisioni dello scrittore di utopia. Per comprenderne l'importanza è stato perciò necessario andare alle radici dell'universo utopico rinascimentale e scandagliarne le motivazioni.

Occorre infine ammettere che, sebbene il concetto di utopia (come società migliore e quindi desiderabile) e il concetto di libertà si siano spesso contraddetti nelle utopie specifiche, vi era nel Rinascimento la possibilità della compresenza di concetti apparentemente contraddittori in uno stesso corpus. La contraddizione va vista come motivo propulsore della storia, a partire da Giordano Bruno, il cui "pensare per contrari" 75 ammette finalmente la compresenza di istanze radicalmente diverse e contradditto-

74 Si veda Alford, Rethinking Freedom. Questo concetto è espresso molto bene anche in Hearfield, Adorno and the Modern Ethos of Freedom, 145: "My contention is that a contradictory politics of truth pervades the modern Ethos of freedom. This contradictory politics emerges as the historically developed opposition of two major strands of thinking of the question of freedom: what I refer to as the opposition of a conceptual ratio and an existential poiesis.... in effect, the conceptual ratio reveals a rationally over-laden articulation of freedom. Here the claim to freedom becomes a sign of unfreedom. Differently yet similarly, the effect of existential poiesis on the claim to freedom is a radical indeterminacy, such that freedom remains indistinguishable from unfreedom. This indicates that both strands of thinking are enmeshed in aporetic claims from which they are unable to extricate themselves.".

75 "il nucleo centrale di questa esperienza (rinascimentale) sta infatti nella dialettica costante - e mai risolta - tra disincanto e utopia, tra sogno e disperazione, tra acuto, e tragico, realismo e una straordinaria capacità di proiettarsi oltre la realtà.... Ignorare uno solo dei lati di questa permanente tensione vuol dire non cogliere il "centro" del Rinascimento, quello che ne ha fatto, e continua a farne, un momento eccezionale nella storia dell'Italia e dell'umanità." Ciliberto, Pensare per contrari, 2. 
rie, e che da tale fruttuosa compresenza il Rinascimento si è arricchito e ha arricchito l'umanità intera. In altre parole, è possibile, data la fluidità del concetto di libertà sospendere la ferrea formulazione del principio di non contraddizione, ed apprendere la difficile convivenza di istanze utopiche, libertà interiore, libertà politica, e libertà di dio nelle utopie italiane del Rinascimento ${ }^{76}$.

\section{UNIVERSITY OF OTTAWA}

\section{OPERE CITATE}

Agostini, Ludovico. La repubblica immaginaria, a cura di Luigi Firpo. Torino: Ramella, 1957.

Alberti, Leon Battista. Momus, o Del Principe, a cura di Giuseppe Martini. Bologna: Zanichelli, 1942.

Alford, C. Fred. Rethinking Freedom. Why Freedom Has Lost Its Meaning and What Can Be Done to Save It. New York: Palgrave McMillan, 2005.

Anonimo. Romanzo di Alessandro, trad. Carlo Franco. Palermo: Sellerio 2005.

Arendt, Hannah. Le origini del totalitarismo, trad. Amerigo Guagnin. Torino: Einaudi, 2004.

Aristotele, Politica, a cura di Carlo Augusto Viano. Milano: Rizzoli, 2002.

Ashcraft, Richard. "Locke's Political Philosophy." The Cambridge Companion to Locke. Cambridge: Cambridge University Press, 1994.

Avineri, Shlomo. "War and Slavery in More's Utopia." International Review of Social History 7:2 (1962): 260-290.

Biavaschi, Paola. "La questione della schiavitù nel diritto romano, alla luce del mito di Atlantide e della città ideale." Alessandria 1 (2007): 79-99.

Boccaccio, Giovanni. Decameron, a cura di Vittore Branca, Torino: UTET, 1956. Campanella, Tommaso. La città del sole. Milano: Feltrinelli, 1987.

Casari, Mario. Alessandro e Utopia nei romanzi persiani medievali. Roma: Bardi, 1999.

Chappell, Vere. "Locke on the Freedom of the Will" in Locke, a cura di Vere Chappell. Oxford: Oxford University Press, 1998, pp. 86-105.

-. "Self-Determination" Early Modern Philosophy. Mind, Matter, and Metaphysics, a cura di Christa Mercer e Eileen O'Neill. Oxford: Oxford University Press, 2005, pp. 141-171.

Hobbes and Bramball on Liberty and Necessity. Cambridge: Cambridge University Press, 1999.

76 Del resto dal punto di vista filosofico anche Emanuele Severino aveva dimostrato il principio di non contraddizione come espresso da Aristotele nella Metafisica presentava una serie di problematiche che potevano essere risolte solo radicalizzandolo. Si veda Severino, "Ritornare a Pamenide" e Il Principio di noncontraddizione. 
Ciliberto, Michele. Pensare per contrari. Disincanto e utopia nel Rinascimento. Roma: Edizioni di Storia e Letteratura, 2005.

Davis, David Brion. The Problem of Slavery in Western Culture. Ithaca: New York, 1966.

de Guevara, Antonio, Reloj de Príncipes. Obras Completas de Fray Antonio de Guevara, a cura di Emilio Blanco. Madrid: Biblioteca Castro, 1994.

Doni, Antonfrancesco. "Il mondo savio e pazzo" in Carlo Curcio, Utopisti italiani del Cinquecento Roma: Colombo, 1944, pp. 64-83.

Eliav-Feldon, Miriam. Realistic Utopias: The Ideal Imaginary Societies of Renaissance, 1516-1630. Oxford: Oxford University Press, 1982.

Grele, Denis. "Relations Économiques Et Rapports Sociaux: Établir Une Cohérence Nationale Au Pays d'Utopie.” Neophilologus 91:1 (2007): 19-32.

Headley, John M. Tommaso Campanella and the Transformation of the World. Princeton: Princeton University Press, 1997.

Hearfield, Colin. Adorno and the Modern Ethos of Freedom. Burlington, VT: Ashgate, 2004.

Honour and Shame: The Values of Mediterranean Society, a cura di John Peristiany. Chicago: University of Chicago Press, 1966.

Huxley, Aldous. Brave New World. London: Hogarth Press, 1984.

Johnson, R. S. More's Utopia: Ideal and Illusion. New Haven: Yale University Press, 1969.

Kant, Immanuel. Fondazione della metafisica dei costumi, trad. Filippo Gonnelli. Bari: Laterza, 2005.

Kosch, Michelle. Freedom and Reason in Kant, Schelling and Kirkegaard. Oxford: Clarendon Press, 2006.

Koselleck, Reinhart. Il vocabolario della modernità, trad. Carlo Sandrelli. Bologna: Il Mulino, 2006.

Kuko, Mislav. "Petri 's Socio-Philosophical Thought Between Realism and Utopianism." Sinthesis Philosophica 22:2 (1996): 410-415.

Langer, Ullrich. Divine and Poetic Freedom in the Renaissance. Princeton: Princeton University Press, 1990.

Levitas, Ruth, The Concept of Utopia, Syracuse: Syracuse University Press, 1991.

Locke, John. An Essay Concerning Human Understanding, a cura di Gary Fuller, Robert Stecker and John P. Wright. London: Routledge, 2000.

Lourde Reyes, Manuel. "La Utopía y el nuevo mundo: El derecho a la esclavitud frente los teólogos juristas españoles." La lámpara de Diógenes: Revista semestral de Filosofía. 6:10-11 (2005): 95-101.

Maravall, José Antonio. "From the Renaissance to the Baroque" in Literature among Discourses, The Spanish Baroque, a cura di Wlad Godzich and Nicholas Spadaccini. Minneapolis, University of Minnesota Press, 1986, pp. 3-40.

Marlin, Randal. "Cartesian Freedom and the Problem of the Mesland Letters" in Essays on Early Modern Philosophers, vol. 1, René Descartes, Parte II, a cura di Vere Chappell. New York: Garland, 1992. (Apparso per la prima volta in Early Modern Philosophy: Metaphysics epistemology and Politics, a cura di Georges J. D. Moyal and Stanley Tweyman Delman. New York: Caravan, 1985, 195-216). 
Meinecke, Friedrich. Lidée de la raison d'Etat dans l'histoire des temps modernes, trad. Maurice Chevallier. Geneve: Droz, 1973.

More Thomas. Utopia, a cura di Luigi Firpo. Napoli: Guida, 1985.

Muccillo, Maria. "Aristotelismo, platonismo ed ermetismo ne «La città felice» di Francesco Patrizi da Cherso." Utopie per gli anni Ottanta. Studi interdisciplinari sui temi, la storia, i progetti, a cura di Giuseppa Saccaro del Buffa e Arthur Lewis, Roma: Gangemi, 1986, pp. 553-579 .

Muccillo, Maria. "Marsilio Ficino e Francesco Patrizi da Cherso." Marsilio Ficino e il ritorno di Platone. 2 voll. Firenze: Leo S. Olschki, 1986, vol., 2, pp. 615-679.

Patrizi, Francesco. "La città felice." Utopisti italiani del Cinquecento, a cura di Carlo Curcio. Bologna: Colombo, 1944.

Perissinotto, Cristina "L'utopia mediterranea di Francesco Patrizi da Cherso," Mediterranoesis: Mediterranean Voices from the Middle Ages and the Renaissance, a cura di Cristina Perissinotto e Roberta Morosini Roma: Salerno, 2007, pp. 151-160.

Piaia, Gregorio. "Entre le mysticisme Platonicien et la 'Philosophie des fleuves'. Le theme des eaux chez Franjo Petri .” Sinthesis Philosophica 22:2 (1996): 381-398.

Quarta, Cosimo, Tommaso Moro. Una reintepretazione dell' "Utopia». Bari: Dedalo, 1991.

Quilligan, Maureen. "Freedom, Service and the Trade in Slaves: the Problem of Labor in Paradise Lost" in Subject and Object in Renaissance Culture, a cura di Margreta De Grazia, Maureen Quilligan e Peter Stallygrass. Cambridge: Cambridge University Press, 1996 , pp. 213-234.

Rabelais, François. Gargantua e Pantagruele, trad. Gildo Passini. Roma: Formiggini, 1925.

Rawls, John. A Theory of Justice. Cambridge, MA: Harvard University Press, 1999. Roseo, Mambrino, "Elogio dei Garamanti" in Carlo Curcio, Utopisti italiani del Cinquecento. Bologna, Colombo, 1944.

Severino, Emanuele. "Ritornare a Pamenide" in Essenza del nichilismo. Brescia: Paideia, 1972, pp. 13-66

Il Principio di non-contraddizione, libro quarto della metafisica di Aristotele. Brescia: La Scuola, 1959.

Vasoli, Cesare. "La critica di Francesco Patrizi alla dottrina aristotelica degli elementi: il fuoco, l'aria e l'acqua." Rinascimento, Seconda Serie, 47 (2007): 93-106.

Zuccolo, Lodovico. La Repubblica d'Evandria e altri dialoghi politici, a cura di R. de Mattei. Roma: Colombo, 1944.

Il Belluzzi, ovvero al città felice, a cura di Amy A. Bernardy. Bologna: Zanichelli, 1929. 